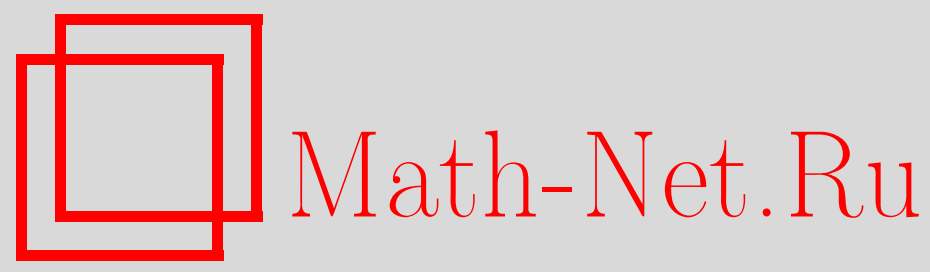

П. С. Дергач, Об однозначности алфавитного декодирования общерегулярных сверхязыков, Дискрет. матем., 2014, том 26, выпуск 1, 32-48

DOI: https://doi.org/10.4213/dm1266

Использование Общероссийского математического портала Math-Net.Ru подразумевает, что вы прочитали и согласны с пользовательским соглашением http://www . mathnet.ru/rus/agreement

Параметры загрузки:

IP : 3.85 .7 .115

26 апреля 2023 г., 13:49:52 


\title{
Об однозначности алфавитного декодирования общерегулярных сверхязыков
}

\author{
() 2014 г. П. С. Дергач ${ }^{1}$
}

\begin{abstract}
Целью работы является установление алгоритмической разрешимости проблемы однозначности алфавитного декодирования общерегулярных сверхязыков.
\end{abstract}

Ключевые слова: однозначное декодирование, общерегулярный сверхъязык, алфавитное кодирование.

\section{1. Введение}

Александром Александровичем Марковым было показано, что проблема однозначности алфавитного декодирования языка $A^{*}$ над заданным конечным алфавитом $A$ алгоритмически разрешима. Он построил также конструктивный алгоритм, доставляющий решение этой проблемы. Доказательство этих фактов можно прочитать, например, в [2].

Автор статьи в своей дипломной работе доказал, что этот результат можно обобщить на случай, когда кодируемое множество слов является регулярным множеством в конечном алфавите $A$. Именно, было показано, что проблема однозначности алфавитного декодирования произвольных регулярных языков над алфавитом $A$ алгоритмически разрешима. Также удалось построить конструктивный алгоритм, доставляющий решение этой проблемы. Доказательство этих фактов можно прочитать в [3].

Здесь продолжается исследование этого направления и рассматривается случай, когда кодируемое множество слов является общерегулярным множеством в конечном алфавите $A$. Тем самым, удается обобщить результат с конечного (по длине рассматриваемых слов) случая на бесконечный. Не ограничивая общности, можно считать, что множество общерегулярных сверхязыков является достаточно широким классом сверхязыков, и именно в нем следует рассматривать проблему однозначности алфавитного декодирования сверхязыков. В статье описывается конструктивный алгоритм, доставляющий решение этой проблемы. Показано также, что проблему однозначности алфавитного декодирования общерегулярных сверхязыков нельзя тривиально свести к проблеме однозначности алфавитного декодирования регулярных языков.

\footnotetext{
${ }^{1}$ Место работы: МГУ им. М. В. Ломоносова e-mail: dergachees@mail.ru
} 


\section{2. Основные понятия и результаты}

Абстрактным конечным автоматом называется набор $V=(A, Q, B, \varphi, \psi)$, где $A, Q, B$ - конечные множества, $\varphi$ - функция, определенная на множестве $Q \times A$ и принимающая значения из $Q, \psi$ - функция, определенная на множестве $Q \times A$ и принимающая значения из $B$. Множества $A, Q, B$ называются соответственно входным алфавитом, алфавитом состояний и выходным алфавитом автомата $V$. Функция $\varphi$ называется функиией переходов, а функция $\psi$ - функиией выходов автомата $V$. Входными словами автомата $V=(A, Q, B, \varphi, \psi)$ называем произвольные конечные последовательности символов алфавита $A$. Для удобства рассматриваем при этом также «пустое» слово, не имеющее ни одного символа и обозначаемое $\Lambda$. Выходными словами алфавита $V$ называем конечные последовательности символов алфавита $B$, словами состояниц - конечные последовательности символов алфавита $Q$ (в обоих случаях допускается и пустое слово $\Lambda$ ). Для каждого состояния автомата $V$ можно рассмотреть набор $(A, Q, B, \varphi, \psi, q)$, определяющий автомат $V$ с выделенным начальным состоянием $q$. Такие наборы $(A, Q, B, \varphi, \psi, q)$ называются инициальными абстрактными конечными автоматами; для них используется обозначение $V_{q}$.

Введем ряд понятий, связанных со словами. Пусть $A-$ некоторое конечное множество. Если $\alpha=a(1) \ldots a(n)$ - конечная последовательность символов $a(1), \ldots, a(n)$ алфавита $A$, то говорим, что $\alpha$ есть слово в алфавите $A$. Число $n$ называем длиной слова $\alpha$ и обозначаем через $|\alpha|$. Длина пустого слова равна 0 . Если $\alpha$ и $\beta$ - слова, причем $\alpha=\beta \beta^{\prime}$ для некоторого слова $\beta^{\prime}$, то говорим, что $\beta-$ начало слова $\alpha, \beta^{\prime}-$ окончание слова $\alpha$. Множество всех слов в алфавите $A$ обозначаем $A^{*}$. Начало слова $\alpha$, имеющее длину $l$, обозначаем $[l(\alpha)$; окончание слова $\alpha$, имеющее длину $l$, обозначаем через $]_{l}(\alpha)$. Обозначим через $\gamma_{l, m}$ слово $]_{m-l}\left(\left[{ }_{m}(\gamma)\right)\right.$, где $|\gamma| \geqslant m>l \geqslant 1$. Также через $\lim (\alpha)$ будем обозначать множество всех букв слова $\alpha$.

Если $\tilde{\alpha}=a(1) a(2) a(3) \ldots$ - бесконечная последовательность символов алфавита $A$, то говорим, что $\tilde{\alpha}$ есть сверхслово в алфавите $A$. Введем ряд понятий, связанных со сверхсловами. Пусть $\alpha-$ конечное слово в алфавите $A$ и $\tilde{\alpha}, \tilde{\alpha}^{\prime}-$ сверхслова в алфавите $A$. Если при этом $\tilde{\alpha}=\alpha \tilde{\alpha^{\prime}}$, то говорим, что $\alpha-$ начало сверхслова $\tilde{\alpha}$, $\tilde{\alpha}^{\prime}$ - окончание сверхслова $\tilde{\alpha}$. Множество всех сверхслов в алфавите $A$ обозначаем $A^{\infty}$. Начало сверхслова $\tilde{\alpha}$, имеющее длину $l$, обозначаем $[l(\tilde{\alpha})$. Окончание сверхслова $\tilde{\alpha}$, полученное отбрасыванием $\left[l_{-1}(\tilde{\alpha}) \text {, будем обозначать через }\right]_{l}(\tilde{\alpha})$. Также вводим обозначение $\tilde{\alpha}_{l, m}$ для слова $]_{m-l}\left(\left[{ }_{m}(\tilde{\alpha})\right)\right.$, где $m>l \geqslant 1$. Будем называть $n p e-$ делом сверхслова $\tilde{\alpha}, \tilde{\alpha} \in A^{\infty}, \tilde{\alpha}=a(1) a(2) \ldots$, множество таких символов $a \in A$, что $a\left(i_{j}\right)=a, j=1,2,3, \ldots$, для некоторой возрастающей последовательности $i_{1}, i_{2}, i_{3}, \ldots$ Предел сверхслова $\tilde{\alpha}$ будем обозначать $\lim (\tilde{\alpha})$.

Доопределим функции переходов и функции выходов автомата $V=$ $(A, Q, B, \varphi, \psi)$ на множество $Q \times A^{*}$ (сохраним за ними те же обозначения). А именно, полагаем по определению

$$
\varphi(q, \Lambda)=q, \varphi(q, \alpha a)=\varphi(\varphi(q, \alpha), a),
$$

где $q \in Q, \alpha \in A^{*}, a \in A$. Аналогично,

$$
\psi(q, \Lambda)=\Lambda, \psi(q, \alpha a)=\psi(\varphi(q, \alpha), a) .
$$

Через $\bar{\psi}: A^{*} \rightarrow B^{*}$ будем обозначать функцию

$$
\bar{\psi}(q, \alpha)=\psi\left(q,\left[{ }_{1}(\alpha)\right) \psi\left(q,\left[{ }_{2}(\alpha)\right) \ldots \psi(q, \alpha) .\right.\right.
$$


Пусть $V_{q}=(A, Q, B, \varphi, \psi, q)$ - инициальный абстрактный конечный автомат, $B^{\prime} \subseteq B$. Множество $M=\left\{\alpha \mid \alpha \in A^{*}, \psi(q, \alpha) \in B^{\prime}\right\}$ называем представимым в абстрактном конечном автомате $V_{q}$ с помощью подмножества $B^{\prime}$ выходных символов. Говорим также, что автомат $V_{q}$ представляет $M$ посредством $B^{\prime}$. Если $\psi(q, \alpha) \in B^{\prime}$, то говорим, что слово $\alpha$ распознается автоматом $V_{q}$ по $B^{\prime}$. Пусть $M \subseteq A^{*} \backslash\{\Lambda\}$. Если существует конечный автомат $V_{q}$, представляющий событие $M$ посредством некоторого подмножества $B^{\prime} \subseteq B$, то событие $M$ называем представимым. Если $B=\{0,1\}, B^{\prime}=\{1\}$, то пишем $M \leftrightarrow V_{q}$.

Недетерминированным конечным автоматом называется набор $V=$ $(A, Q, B, \gamma)$, где $A, Q, B$ - конечные множества, $\gamma$ - функция, определенная на множестве $Q \times A$ и принимающая в качестве своих значений подмножества множества $Q \times B$. Если для каждой пары $(q, a)$, где $q \in Q$ и $a \in A$, значение $\gamma(q, a)$ есть одноэлементное множество $\left(q^{\prime}, b\right)$, то можно определить функции $\varphi, \psi: \varphi(q, a)=q^{\prime}, \psi(q, a)=b$. При этом возникает некоторый абстрактный конечный автомат $V=(A, Q, B, \varphi, \psi)$. В указанном смысле абстрактный конечный автомат можно рассматривать как частный случай недетерминированного конечного автомата. Понятие инициального недетерминированного конечного автомата, аналогичное понятию инициального абстрактного конечного автомата, возникает, если выделено некоторое подмножество $Q^{\prime} \subseteq Q$ начальных состояний автомата. Пусть $Q^{\prime}$ - такое выделенное подмножество и $\alpha=a(1) \ldots a(s)-$ слово в алфавите $A$. Определим класс $\tilde{\gamma}\left(Q^{\prime}, \alpha\right)$ последовательностей $(q(1), b(1)), \ldots,(q(s), b(s))$. Каждая последовательность этого класса удовлетворяет следующим условиям:

1) $q(1) \in Q^{\prime}$

2) $(q(i+1), b(i)) \in \gamma(q(i), a(i)), i=1, \ldots, s$;

(в случае $i=s$ рассматривается значение $q(s+1)$, не включаемое в последовательность $\left.\tilde{\gamma}\left(Q^{\prime}, \alpha\right)\right)$.

Недетерминированный инициальный автомат $V=\left(A, Q, B, \gamma, Q^{\prime}\right)$ можно рассматривать как модель устройства, применяемого для распознавания входных последовательностей. Аналогично случаю обычного конечного автомата, при этом выделяется подмножество $B^{\prime}$ множества $B$ выходных символов. Множество входных слов $M$, распознаваемых автоматом, состоит из тех и только тех слов $\alpha \in A^{*}$, для которых в $\tilde{\gamma}\left(Q^{\prime}, \alpha\right)$ существует последовательность $(q(1), b(1)), \ldots,(q(s), b(s))$, удовлетворяющая условию $b(s) \in B^{\prime}$. Говорим также, что множество $M$ представимо в недетерминированном конечном автомате $V$ с помощью подмножества $B^{\prime}$ въходных символов.

Пусть $V_{q}=(A, Q, B, \varphi, \psi, q)$ - инициальный абстрактный конечный автомат. Для произвольного сверхслова $\tilde{\alpha}=a(1) a(2) a(3) \ldots$ в алфавите $A$ обозначаем через $\tilde{\psi}(q, \tilde{\alpha})$ такое сверхслово $\tilde{\beta}=b(1) b(2) b(3) \ldots$, что $b(i)=\psi(q, a(1) \ldots a(i)), i=1,2,3, \ldots$ Пусть $B_{1}, \ldots, B_{k}$ - некоторые попарно различные подмножества множества $B$. Множество

$$
M=\left\{\tilde{\alpha} \mid \tilde{\alpha} \in A^{\infty}, \lim (\tilde{\psi}(q, \tilde{\alpha})) \in\left\{B_{1}, B_{2}, \ldots, B_{k}\right\}\right\}
$$

называем представимым в автомате $V_{q}$ с помощъю семейства $\left\{B_{1}, \ldots, B_{k}\right\}$ подмножеств выходных символов. Подмножества множества $A^{\infty}$ называем сверхсобътиями в алфавите $A$. Сверхсобытие $S$ называется представимым, если существует абстрактный конечный инициальный автомат $V_{q}=(A, Q, B, \varphi, \psi, q)$, в котором это сверхсобытие представимо с помощью некоторого семейства $B^{\prime}$ подмножеств выходных символов. Говорим в последнем случае, что автомат $V_{q}$ представляет сверхсобытие $S$ посредством $B^{\prime}$. Пишем $S \stackrel{B^{\prime}}{\leftrightarrow}>V_{q}$. 
Пусть $A=\left\{a_{1}, \ldots, a_{r}\right\}-$ произвольный конечный непустой алфавит. Пусть $P_{1}, P_{2}$ - непустые множества слов в алфавите $A$. Здесь и далее для удобства пустое слово не считается элементом множества $A^{*}$. Определим следующие операции над $P_{1}$ и $P_{2}$ :

1) произведение множеств $P_{1}$ и $P_{2}$ (обозначаем $P_{1} \cdot P_{2}$ ) есть множество всех слов вида $\alpha_{1} \alpha_{2}$, где $\alpha_{1} \in P_{1}, \alpha_{2} \in P_{2}$;

2) итерация множества $P_{1}$ (обозначаем $\left(P_{1}\right)^{*}$ ) есть множество всех слов вида $\alpha_{1} \ldots \alpha_{k}$, где $\alpha_{1} \in P_{1}, \ldots, \alpha_{k} \in P_{1}, k \geqslant 1$.

Введем понятие регулярного множества в алфавите $A$. Множество $P \subseteq A^{*}$ называем регулярным в алфавите $A$, если его можно получить из множеств вида $\{a\}, a \in A$, применением конечного числа операций $\cup, \cdot,()^{*}$. Более подробно, определение регулярных множеств таково:

1) $\{a\}$, где $a$ - произвольная буква алфавита $A$, - регулярное множество в алфавите $A$;

2) если $P_{1}, P_{2}$ - регулярные множества в алфавите $A$, то $P_{1} \cup P_{2}, P_{1} \cdot P_{2},\left(P_{1}\right)^{*}$ - регулярные множества в алфавите $A$;

$3)$ регулярность произвольного множества в алфавите $A$ устанавливается в соответствиями с пп. 1), 2) за конечное число шагов.

Рассмотрим следующие две операции, позволяющие получать сверхсобытия:

1) произведение события $M_{1}$ в алфавите $A$ и сверхсобытия $M_{2}$ в этом же алфавите (обозначается $M_{1} \cdot M_{2}$ ) есть сверхсобытие в алфавите $A$, состоящее из всех таких сверхслов $\tilde{\alpha}=a(1) a(2) \ldots$, что при некотором $i \in\{1,2, \ldots\}$ слово $a(1) \ldots a(i)$ принадлежит $M_{1}$, а сверхслово $a(i+1) a(i+2) \ldots$ принадлежит $M_{2}$.

2) Сверхитерация события $M$ в алфавите $A$ (обозначается $M^{\infty}$ ) есть сверхсобытие в алфавите $A$, состоящее из всех таких сверхслов $\tilde{\alpha}=a(1) a(2) \ldots$, что $a\left(i_{j}\right) a\left(i_{j}+1\right) \ldots a\left(i_{j+1}-1\right) \in M, j=1,2, \ldots$, для некоторой возрастающей последовательности $i_{1}=1, i_{2}, i_{3}, \ldots$ натуральных чисел.

Определим класс общерегулярных сверхсобытий в алфавите $A$ :

1 ) если $R$ - регулярное событие в алфавите $A$, то $R^{\infty}$ есть общерегулярное сверхсобытие в алфавите $A$;

2) если $R_{1}$ - регулярное событие в алфавите $A$ и $R_{2}$ - общерегулярное сверхсобытие в алфавите $A$, то $R_{1} \cdot R_{2}$ есть общерегулярное сверхсобытие в алфавите $A$;

$3)$ если $R_{1}$ и $R_{2}$ - общерегулярные сверхсобытия в алфавите $A$, то $R_{1} \cup R_{2}-$ общерегулярное сверхсобытие в алфавите $A$;

4) общерегулярность произвольного сверхсобытия в алфавите $A$ устанавливается в соответствии с пп. 1),2),3) за конечное число шагов.

Зафиксируем два конечных непустых алфавита $A=\left\{a_{1}, a_{2}, \ldots, a_{r}\right\}$ и $B$. Пусть $\beta_{1}, \beta_{2}, \ldots, \beta_{r}$ - слова в алфавите $B$. Некоторые из этих слов (и даже все) могут совпадать. Рассмотрим отображение $f: A \rightarrow B^{*}$ :

$$
f\left(a_{1}\right)=\beta_{1}, f\left(a_{2}\right)=\beta_{2}, \ldots, f\left(a_{r}\right)=\beta_{r} .
$$

Это соотношение называется схемой кодирования. Доопределим отображение $f$ до отображения $\tilde{f}: A^{\infty} \rightarrow B^{\infty}$ следующим образом:

$$
\tilde{f}\left(a_{i_{1}} a_{i_{2}} \ldots a_{i_{n}} \ldots\right)=\beta_{i_{1}} \beta_{i_{2}} \ldots \beta_{i_{n}} \ldots
$$

Отображение $\tilde{f}$ будем называть алфавитным кодированием из алфавита $A$ в алфавит B. 
Пусть есть некоторое сверхрегулярное множество $\tilde{P}$ в алфавите $A$ и некоторое алфавитное кодирование $\tilde{f}$. Пусть $\tilde{\beta} \in \tilde{f}(\tilde{P})$. Тогда $\tilde{\alpha} \in \tilde{P}$ называется расшифровкой $\tilde{\beta}$ при алфавитном кодировании $\tilde{f}$ на сверхрегулярном множестве $\tilde{P}$ или расшифровкой $\tilde{\beta}$, если $\tilde{f}(\tilde{\alpha})=\tilde{\beta}$. Таких расшифровок может быть несколько. Если $\tilde{f}\left(\tilde{\alpha_{1}}\right) \neq \tilde{f}\left(\tilde{\alpha_{2}}\right)$ для любых различных $\tilde{\alpha_{1}}, \tilde{\alpha_{2}} \in \tilde{P}$, то говорим, что декодирование однозначно на $\tilde{P}$ по $\tilde{f}$.

Теорема 1. Существует алгоритм, определяющий по произвольной паре $(\tilde{f}, \tilde{R})$, где $\tilde{f}$ - алфавитное кодирование из алфавита $A$ в алфавит $B$, а $\tilde{R}$ - сверхрегулярное множество в алфавите $A$, однозначно ли декодирование на $\tilde{R}$ по $\tilde{f}$.

\section{3. Доказательства вспомогательных утверждений}

Теорема Клини. Множество слов является представимым тогда и только тогда, когда оно регулярно.

Теорема Мак-Ноттона. Множество сверхслов является представимым тогда и только тогда, когда оно общерегулярно.

Лемма о детерминизации. Любой недетерминированный конечный автомат $V$ c q состояниями может быть преобразован в абстрактный конечный автомат $V^{\prime}$ с не более чем $2^{q}$ состояниями так, что представляемые этими автоматами по $\{1\}$ множества совпадают.

Доказательства этих утверждений можно найти в [1].

Лемма об алфавитной детерминизации. Пусть $V_{q}=(A, Q,\{0,1\}, \varphi, \psi, q)$ инициальный абстрактный конечный автомат, представляющий событие $M$ посредством $\{1\}$. Пусть $\tilde{f}$ - алфавитное кодирование из алфавита $A=\left\{a_{1}, \ldots, a_{r}\right\}$ в алфавит $B,|f|=\max _{i=1, \ldots, r}\left|\tilde{f}\left(a_{i}\right)\right|$. Тогда событие

$$
\tilde{f}(M):=\{\beta \in B \mid \exists \alpha \in M: \tilde{f}(\alpha)=\beta\}
$$

представимо посредством $\{1\}$ некоторым инициальным абстрактным конечным автоматом $V_{q}^{\prime}=\left(B, Q^{\prime},\{0,1\}, \varphi^{\prime}, \psi^{\prime}, q^{\prime}\right)$ с не более чем $2^{|Q|+r|Q|(|f|-1)}$ состояниями.

Доказательство. Заменим в автомате $V_{q}$ входной алфавит $A$ алфавитом $\tilde{f}(A):=$ $\bigcup_{i=1}^{r}\left\{\tilde{f}\left(a_{i}\right)\right\}$ и соответственно поправим функции перехода и выхода, заменив в них все $a_{i}$ на $\tilde{f}\left(a_{i}\right)$. Выходной алфавит, алфавит состояний и начальное состояние оставим теми же. Полученный автомат $V_{q}^{\prime}$ может оказаться недетерминированным, так как некоторые буквы нового входного алфавита в функциях перехода и выхода могут встречаться больше одного раза для одного и того же состояния. Тем не менее, $V_{q}^{\prime}$ представляет событие $\tilde{f}(M)$ посредством $\{1\}$.

Теперь заменим для каждой пары состояний $q_{l}, q_{m}$ и каждого слова $\tilde{f}\left(a_{i}\right)$ имеющиеся в $V_{q}^{\prime}$ переходы из состояния $q_{l}$ в состояние $q_{m}$ по слову $\tilde{f}\left(a_{i}\right)=\beta_{1} \ldots \beta_{s}$ последовательностью состояний с побуквенными переходами $\beta_{1}, \ldots, \beta_{s}$ так, как это изображено на рисунке 1: 

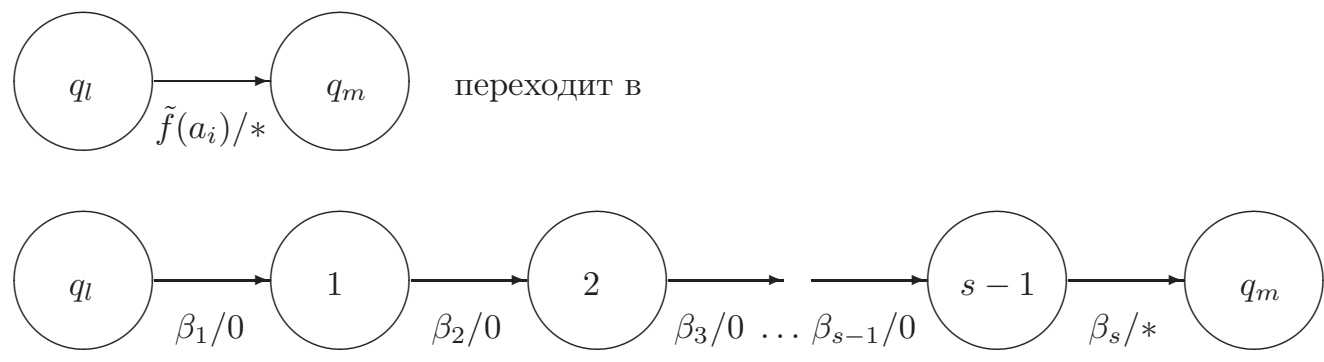

рис. 1

Полученный недетерминированный конечный автомат $V_{q}^{\prime \prime}$ по-прежнему представляет событие $\tilde{f}(M)$ посредством $\{1\}$, имеет входной алфавит $B$, выходной алфавит $\{0,1\}$ и множество состояний $Q^{\prime}$, по мощности не превосходящее $|Q|+r|Q|(|f|-1)$. В самом деле, общее количество переходов в автомате $V_{q}^{\prime}$ равно $r|Q|$ и каждый из них добавляет к общему числу состояний автомата $V_{q}^{\prime \prime}$ не более $|f|-1$ состояний.

Осталось применить лемму о детерминизации к автомату $V_{q}^{\prime \prime}$. Полученный абстрактный конечный автомат $\tilde{V}_{q}$ имеет входной алфавит $B$, выходной алфавит $\{0,1\}$, представляет событие $\tilde{f}(M)$ посредством $\{1\}$ и имеет не более $2^{|Q|+r|Q|(|f|-1)}$ состояний. Утверждение леммы доказано.

Лемма о сверхсобытии. Пусть $R$ - общерегулярное сверхсобытие в алфавите $A$. Тогда существуют такие регулярные события $S_{1}, M_{1}, \ldots, S_{k}, M_{k}$ в алфавите $A$, чmо $R=\bigcup_{i=1}^{k} S_{i} \cdot\left(M_{i}\right)^{\infty}$.

Доказательство. Каждое общерегулярное сверхсобытие можно получить за конечное число шагов используя три следующих правила:

$1)$ если $R$ - регулярное событие в алфавите $A$, то $R^{\infty}$ есть общерегулярное сверхсобытие в алфавите $A$;

2) если $R_{1}$ - регулярное событие в алфавите $A$ и $R_{2}$ - общерегулярное сверхсобытие в алфавите $A$, то $R_{1} \cdot R_{2}$ есть общерегулярное сверхсобытие в алфавите $A$;

3 ) если $R_{1}$ и $R_{2}$ - общерегулярные сверхсобытия в алфавите $A$, то $R_{1} \cup R_{2}$ - общерегулярное сверхсобытие в алфавите $A$.

Докажем утверждение леммы индукцией по длине минимального вывода из правил 1),2),3) общерегулярного сверхсобытия $R$.

База индукиии. Если минимальная длина вывода равна 1, то общерегулярное сверхсобытие $R$ получено по правилу 1 ) и имеет вид $R^{\infty}$ для некоторого регулярного события $R$ в алфавите $A$.

Индуктивный переход. Пусть утверждение справедливо для всех общерегулярных сверхсобытий с минимальной длиной вывода не больше $n$. Рассмотрим общерегулярное сверхсобытие $R$ с минимальной длиной вывода $n+1$. Если бы последним правилом в этом выводе было правило 1), то длина вывода была бы равна 1 , что невозможно. Если последним правилом было правило 2$)$, то $R=R_{1} \cdot R_{2}$ для некоторого регулярного события $R_{1}$ и общерегулярного сверхсобытия $R_{2}$ с минимальной длиной вывода $n$. По предположению индукции, существуют такие регулярные со- 
бытия $S_{1}, M_{1}, \ldots, S_{k}, M_{k}$, что $R_{2}=\bigcup_{i=1}^{k} S_{i} \cdot\left(M_{i}\right)^{\infty}$. Поэтому

$$
R=R_{1} \cdot R_{2}=R_{1} \cdot\left(\bigcup_{i=1}^{k} S_{i} \cdot\left(M_{i}\right)^{\infty}\right)=\bigcup_{i=1}^{k}\left(R_{1} \cdot S_{i}\right) \cdot\left(M_{i}\right)^{\infty}
$$

Так как для всех $i=1, \ldots, k$ множества $R_{1} \cdot S_{i}$ регулярны, то это - искомое представление. Если же последним правилом было правило 3 ), то $R=R_{1} \cup R_{2}$ для некоторых общерегулярных сверхсобытий $R_{1}$ и $R_{2}$ с минимальной длиной вывода не больше $n$. По предположению индукции существуют такие регулярные события $S_{1}, M_{1}, \ldots, S_{k}, M_{k}, P_{1}, T_{1}, \ldots, P_{m}, T_{m}$, что

$$
R_{1}=\bigcup_{i=1}^{k} S_{i} \cdot\left(M_{i}\right)^{\infty} \quad \text { и } \quad R_{2}=\bigcup_{i=1}^{m} P_{i} \cdot\left(T_{i}\right)^{\infty} .
$$

Поэтому

$$
R=R_{1} \cup R_{2}=\left(\bigcup_{i=1}^{k} S_{i} \cdot\left(M_{i}\right)^{\infty}\right) \cup\left(\bigcup_{i=1}^{m} P_{i} \cdot\left(T_{i}\right)^{\infty}\right) .
$$

Это искомое представление. Утверждение леммы доказано.

Лемма о сокращении. Пусть $A-$ конечный алфавит, $\delta_{1}, \delta_{2}, \xi_{1}, \xi_{2}, \beta-$ слова (возможно, пустые) в алфавите $A$, пусть $P_{1}, P_{2}$ - регулярные множества в алфавите $A$ u $\delta_{1} \beta \delta_{2} \in P_{1}, \xi_{1} \beta \xi_{2} \in P_{2}$, nycms $V_{1}=\left(A, Q_{1},\{0,1\}, \varphi_{1}, \psi_{1}, q_{1}\right), V_{2}=$ $\left(A, Q_{2},\{0,1\}, \varphi_{2}, \psi_{2}, q_{2}\right)$ - инициальные абстрактные конечные автоматы, представляющие по множеству $\{1\}$ множества $P_{1}, P_{2}$ соответственно. Тогда существует такое слово $\beta^{\prime}$ в алфавите $A,\left|\beta^{\prime}\right| \leqslant\left|Q_{1}\right|\left|Q_{2}\right|$, что $\delta_{1} \beta^{\prime} \delta_{2} \in P_{1}, \xi_{1} \beta^{\prime} \xi_{2} \in P_{2}$.

Доказательство. Допустим, что $|\beta|>\left|Q_{1}\right|\left|Q_{2}\right|$. Рассмотрим такое множество $T$ пар состояний автоматов $V_{1}, V_{2}$, что $T=\left\{\left(\varphi_{1}\left(q_{1}, \delta_{1}[l(\beta)), \varphi_{2}\left(q_{2}, \xi_{1}\left[{ }_{l}(\beta)\right)\right), 1 \leqslant l \leqslant|\beta|\right\}\right.\right.$. Так как $|T|<\left|Q_{1}\right|\left|Q_{2}\right|+1$, то существуют такие $1 \leqslant l_{1}<l_{2} \leqslant\left|Q_{1}\right|\left|Q_{2}\right|+1$, что $\varphi_{1}\left(q_{1}, \delta_{1}\left[l_{1}(\beta)\right)=\varphi_{1}\left(q_{1}, \delta_{1}\left[l_{2}(\beta)\right), \varphi_{2}\left(q_{2}, \xi_{1}\left[l_{1}(\beta)\right)=\varphi_{2}\left(q_{2}, \xi_{1}\left[l_{2}(\beta)\right)\right.\right.\right.\right.$. Пусть $\beta_{1}$ и $\beta_{2}$ определяются равенствами $\beta_{1}=\left[l_{1}(\beta), \beta=\left[l_{2}(\beta) \beta_{2}\right.\right.$. Тогда

$$
\begin{gathered}
\psi_{1}\left(q_{1}, \alpha_{1} \beta_{1} \beta_{2} \alpha_{2}\right)=\psi_{1}\left(\varphi_{1}\left(q_{1}, \alpha_{1} \beta_{1}\right), \beta_{2} \alpha_{2}\right)=\psi_{1}\left(\varphi_{1}\left(q_{1}, \alpha_{1}\left[l_{2}(\beta)\right), \beta_{2} \alpha_{2}\right)=\right. \\
=\psi_{1}\left(\varphi_{1}\left(q_{1}, \delta_{1}\left[l_{2}(\beta) \beta_{2}\right), \delta_{2}\right)=\psi_{1}\left(\varphi_{1}\left(q_{1}, \delta_{1} \beta\right), \delta_{2}\right)=\psi_{1}\left(q_{1}, \delta_{1} \beta \delta_{2}\right)=1 .\right.
\end{gathered}
$$

Аналогично $\psi_{2}\left(q_{2}, \xi_{1} \beta_{1} \beta_{2} \xi_{2}\right)=1$. Таким образом, существует такое $\beta^{\prime \prime}=\beta_{1} \beta_{2}$, что $\delta_{1} \beta^{\prime \prime} \delta_{2} \in P_{1}, \xi_{1} \beta^{\prime \prime} \xi_{2} \in P_{2}$ и $\left|\beta^{\prime \prime}\right|<|\beta|$. Проведя конечное число раз процедуру сокращения $\beta$, получим такое $\beta^{\prime}$ длины не больше $\left|Q_{1}\right|\left|Q_{2}\right|$, что $\delta_{1} \beta^{\prime} \delta_{2} \in P_{1}, \xi_{1} \beta^{\prime} \xi_{2} \in P_{2}$. Утверждение леммы доказано.

Лемма о разрезе. Пусть $\alpha-$ слово в алфавите $A, P$ - регулярное множество в алфавите $A$, представимое с помощью инициального абстрактного конечного автомата $V_{q_{0}}=\left(A, Q,\{0,1\}, \varphi, \psi, q_{0}\right)$ с помощью множества $\{1\}, \alpha \in P, 1 \leqslant s<|\alpha|$. Тогда существуют регулярные множества $P_{1}$ и $P_{2}$ в алфавите $A$, представимье $c$ помощъю инициальных абстрактных конечных автоматов с входным алфавитом $A$, выходным алфавитом $\{0,1\}$ и алфавитом состояний $|Q|$ с помощъю множества $\{1\}$ удовлетворяющие условиям $P_{1} \cdot P_{2} \subseteq P, \alpha \in P_{1} \cdot P_{2},\left[{ }_{s}(\alpha) \in P_{1},\right]_{|\alpha|-s}(\alpha) \in P_{2}$. 
Доказательство. Пусть $\varphi\left(q_{0},[s(\alpha))=q_{1}\right.$. Рассмотрим два инициальных абстрактных конечных автомата $V_{1}=\left(A, Q,\{0,1\}, \varphi, \psi_{1}, q_{0}\right), V_{2}=\left(A, Q,\{0,1\}, \varphi, \psi, q_{1}\right)$, где $\psi_{1}(q, a)=1$ при $\varphi(q, a)=q_{1}$ и $\psi_{1}(q, a)=0$ в противном случае. По теореме Клини эти два автомата по множеству $\{1\}$ представляют регулярные множества $P_{1}$ и $P_{2}$. Так как $\varphi\left(q_{0},{ }_{s}(\alpha)\right)=q_{1}$, то $\psi_{1}\left(q_{0},{ }_{s}(\alpha)\right)=1$, т. е. $\left[{ }_{s}(\alpha) \in P_{1}\right.$. Далее, так как $\alpha \in P$, то

$$
\left.\left.1=\psi\left(q_{0}, \alpha\right)=\psi\left(\varphi\left(q_{0},\left[{ }_{s}(\alpha)\right),\right]_{|\alpha|-s}(\alpha)\right)=\psi\left(q_{1},\right]_{|\alpha|-s}(\alpha)\right),\right]_{|\alpha|-s}(\alpha) \in P_{2} .
$$

Значит, $\alpha \in P_{1} \cdot P_{2}$.

Пусть $\alpha_{1} \in P_{1}, \alpha_{2} \in P_{2}$. Тогда $\varphi\left(q_{0}, \alpha_{1}\right)=q_{1}, \psi\left(q_{1}, \alpha_{2}\right)=1$. Значит, $\psi\left(q_{0}, \alpha_{1} \alpha_{2}\right)=$ $\psi\left(\varphi\left(q_{0}, \alpha_{1}\right), \alpha_{2}\right)=\psi\left(q_{1}, \alpha_{2}\right)=1$, т. е. $\alpha_{1} \alpha_{2} \in P$. Поэтому $P_{1} \cdot P_{2} \subseteq P$. Утверждение леммы доказано.

Лемма о начале сверхсобытия. Существует алгоритм, которьй по произволъному натуральному $k$ и произвольному общерегулярному сверхсобытию $\tilde{R}$ в алфавите A строит множество

$$
M(k, \tilde{R}):=\left\{\alpha \in A^{k} \mid \exists \gamma \in A^{\infty}: \alpha \gamma \in \tilde{R}\right\}
$$

Доказательство. Рассмотрим произвольное натуральное $k$ и произвольное общерегулярное сверхсобытие $\tilde{R}$ в алфавите $A$. По теореме Мак-Ноттона $\tilde{R}$ представимо некоторым абстрактным конечным инициальным автоматом $V_{q_{1}}=\left(A, Q, B, \varphi, \psi, q_{1}\right)$ с помощью множества $B^{\prime} \subseteq B$. Возьмем произвольное $\alpha \in A^{k}$ и проверим, верно ли, что $\alpha \in M(k, \tilde{R})$.

Пусть $Q=\left\{q_{1}, \ldots, q_{n}\right\}$. Пусть $1 \leqslant i \leqslant n$ и $\beta \in A^{*}, \varphi\left(q_{i}, \beta\right)=q_{i}, \lim \left(\bar{\psi}\left(q_{i}, \beta\right)\right)=$ $B_{0} \in B^{\prime}$. Более точно, пусть

$$
\psi\left(q_{i},{ }_{p_{1}}(\beta)\right) \bigcup \psi\left(q_{i},{ }_{p_{2}}(\beta)\right) \bigcup \ldots \bigcup \psi\left(q_{i},\left[{ }_{p}(\beta)\right)=B_{0},\right.
$$

где $s=\left|\lim \left(B_{0}\right)\right|$. Другими словами, мы берем минимальный по мощности набор букв слова $\beta$, во время подачи которых автомат $V_{q_{1}}$ выдает те буквы, которые образуют $B_{0}$. Введем обозначения:

$$
\begin{aligned}
& \varphi\left(q_{i},{ }_{p_{1}-1}(\beta)\right):=q_{1}^{s t}, \varphi\left(q_{i},{ }_{p_{1}}(\beta)\right):=q_{1}^{f i}, \\
& \varphi\left(q_{i},{ }_{p_{2}-1}(\beta)\right):=q_{2}^{s t}, \quad \varphi\left(q_{i},{ }_{p_{2}}(\beta)\right):=q_{2}^{f i}, \\
& \ldots \\
& \varphi\left(q_{i},{ }_{p_{s}-1}(\beta)\right):=q_{s}^{s t}, \quad \varphi\left(q_{i},\left[{ }_{p_{s}}(\beta)\right):=q_{s}^{f i} .\right.
\end{aligned}
$$

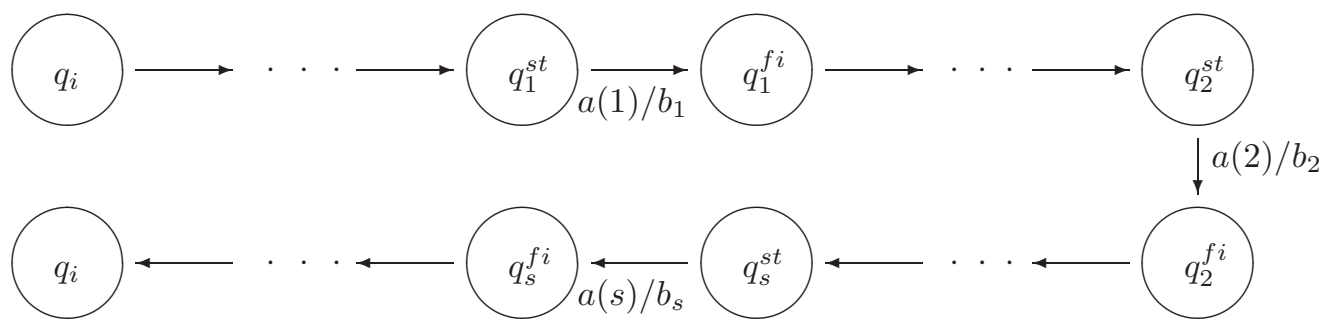

рис. 2 
Здесь $\left\{b_{1}, b_{2}, \ldots, b_{s}\right\}=B_{0}$ и некоторые состояния могут повторяться. Так как $\varphi\left(q_{i},\left[p_{1}-1(\beta)\right)=q_{1}^{s t}\right.$, то, выкидывая из слова $\left[p_{1}-1(\beta)\right.$ все куски, образующие циклы в диаграмме переходов, получаем такое подслово $\beta_{1}$ слова $\left[p_{1}-1(\beta)\right.$, что $\varphi\left(q_{i}, \beta_{1}\right)=q_{1}^{\text {st }}$ и $\left|\beta_{1}\right| \leqslant n-1$. Аналогично можно найти такое слово $\beta_{2}$, что $\varphi\left(q_{1}^{f i}, \beta_{2}\right)=q_{2}^{s t}$ и $\left|\beta_{2}\right| \leqslant n-1$. Действуя таким образом и дальше, получаем слово $\gamma:=\beta_{1} a(1) \beta_{2} a(2) \ldots a(s) \beta_{s+1}$, для которого $\varphi\left(q_{i}, \gamma\right)=q_{i}$. При этом $|\gamma| \leqslant(s+1)(n-1)+s \leqslant(|B|+1)(n-1)+|B|$. Обозначим это число через $L$.

Теперь легко проверить, верно ли, что $\alpha \in M(k, \tilde{R})$. Это верно тогда и только тогда, когда в диаграмме переходов автомата $V_{q_{1}}$ существует состояние $\tilde{q}$, достижимое из состояния $\varphi\left(q_{1}, \alpha\right)$, и для некоторого слова $\beta$ в алфавите $A$ имеем $\varphi(\tilde{q}, \beta)=\tilde{q}$, $\lim (\bar{\psi}(\tilde{q}, \beta)) \in B^{\prime}$. По доказанному выше, достаточно проверить слова $\beta \in A^{L}$, что можно сделать в силу конечности множества $A^{L}$.

Осталось для всех слов $\alpha \in A^{k}$ проверить, верно ли, что $\alpha \in M(k, \tilde{R})$. Таким образом мы узнаем все элементы множества $M(k, \tilde{R})$. Утверждение леммы доказано.

Главная лемма. Пусть $S_{1}, M_{1} \ldots, S_{k}, M_{k}$ - регулярные события в алфавите $A$, которые можно представить абстрактными конечными инициальными автоматами с входным алфавитом $A$, выходным алфавитом $B$ и алфавитами состояний мощности не более $Q$. Пусть алфавитное кодирование $\tilde{f}$ из алфавита $A$ в алфавит $B$ не однозначно декодируемо на сверхсобытии $\tilde{R}=\bigcup_{i=1}^{k} S_{i} \cdot\left(M_{i}\right)^{\infty}$. Пусть $\tilde{Q}:=2^{Q+Q|A|(|f|-1)}$. Тогда для некоторых $1 \leqslant j \leqslant k, 1 \leqslant h \leqslant k$ существуют такие слова $\alpha_{1} \in S_{j}, \alpha_{2} \in S_{h}, \beta_{1} \in M_{j}^{*}, \beta_{2} \in M_{h}^{*}$, что:

1) $\alpha_{1} \cdot\left(\beta_{1}\right)^{\infty} \neq \alpha_{2} \cdot\left(\beta_{2}\right)^{\infty}$,

2) $\tilde{f}\left(\alpha_{1} \cdot\left(\beta_{1}\right)^{\infty}\right)=\tilde{f}\left(\alpha_{2} \cdot\left(\beta_{2}\right)^{\infty}\right)$,

3) $\max \left(\left|\alpha_{1}\right|+\left|\beta_{1}\right|,\left|\alpha_{2}\right|+\left|\beta_{2}\right|\right) \leqslant$

$\leqslant 3 Q^{2}+1+\tilde{Q}^{2}(\tilde{Q}+3)+2 \tilde{Q}^{2}(\tilde{Q}+3)\left(\frac{|B|^{\tilde{Q}^{2}(\tilde{Q}+3)}-|B|}{|B|-1}\right)$.

Доказательство. Так как $\tilde{f}$ не однозначно декодируемо на $\tilde{R}$, то существуют такие $\tilde{\alpha_{1}}, \tilde{\alpha_{2}} \in \tilde{R}$, что $\tilde{\alpha_{1}} \neq \tilde{\alpha_{2}}, \tilde{f}\left(\tilde{\alpha_{1}}\right)=\tilde{f}\left(\tilde{\alpha_{2}}\right)$. Без нарушения общности можно считать, что $\tilde{\alpha_{1}} \in S_{1} \cdot\left(M_{1}\right)^{\infty}, \tilde{\alpha_{2}} \in S_{2} \cdot\left(M_{2}\right)^{\infty}$. Случай, когда $\tilde{\alpha_{1}}, \tilde{\alpha_{2}} \in S_{1} \cdot\left(M_{1}\right)^{\infty}$, разбирается аналогично. Тогда существуют такие $s_{1} \in S_{1}, s_{2} \in S_{2}, m_{1}^{i} \in M_{1}, m_{2}^{i} \in M_{2}(i=$ $1, \ldots, \infty)$, что $\tilde{\alpha_{1}}=s_{1} \cdot m_{1}^{1} \cdot m_{1}^{2} \ldots, \tilde{\alpha_{2}}=s_{2} \cdot m_{2}^{1} \cdot m_{2}^{2} \ldots$ Введем обозначения:

$$
\begin{gathered}
l:=\max _{s \in \mathbb{N}}\left\{s \mid{ }_{s}\left(\alpha_{1}\right)={ }_{s}\left(\alpha_{2}\right)\right\}, \alpha:={ }_{l}\left(\alpha_{1}\right), \\
\left.\left.a_{1}:=\left(\alpha_{1}\right)_{l, l+1}, a_{2}:=\left(\alpha_{1}\right)_{l, l+1}, \tilde{\beta}:=\right]_{l+1}\left(\alpha_{1}\right), \tilde{\gamma}:=\right]_{l+1}\left(\alpha_{2}\right) .
\end{gathered}
$$

Рассмотрим 4 случая.

Случай 1.

$l+1 \leqslant\left|s_{1}\right|, l+1 \leqslant\left|s_{2}\right|$ (см. рис. 3 ). 

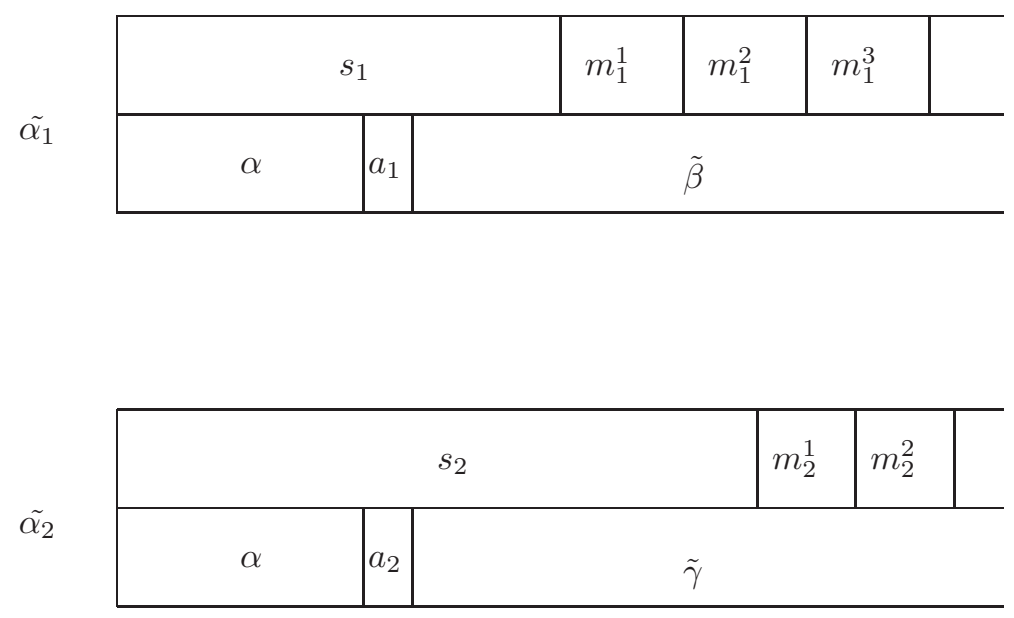

рис. 3

Применим лемму о разрезе для слов $s_{1} \in S_{1}$ и $s_{2} \in S_{2}$ по местам $s:=l$ и $s:=l+1$ так, как это показано на рис. 4.

\begin{tabular}{|c|c|c|c|c|c|c}
\hline$\tilde{\alpha}_{1}$ & $s_{1}^{2}$ & $s_{1}^{3}$ & $m_{1}^{1}$ & $m_{1}^{2}$ & $m_{1}^{3}$ & \\
\cline { 2 - 8 } & $a_{1}$ & \multicolumn{6}{|c}{$\tilde{\beta}$} \\
\hline
\end{tabular}

\begin{tabular}{|c|c|c|c|c|}
\hline$s_{2}^{1}$ & $s_{2}^{2}$ & $s_{2}^{3}$ & $m_{2}^{1}$ & $m_{2}^{2}$ \\
\hline$\alpha$ & $a_{2}$ & & & \\
\hline
\end{tabular}

рис. 4

Здесь $s_{1}^{1} \in S_{1}^{1}, s_{1}^{2} \in S_{1}^{2}, s_{1}^{3} \in S_{1}^{3}, s_{2}^{1} \in S_{2}^{1}, s_{2}^{2} \in S_{2}^{2}, s_{2}^{3} \in S_{2}^{3}, S_{1}^{1} \cdot S_{1}^{2} \cdot S_{1}^{3} \subseteq S_{1}$, $S_{2}^{1} \cdot S_{2}^{2} \cdot S_{2}^{3} \subseteq S_{2}$ и каждое из событий $S_{1}^{1}, S_{1}^{2}, S_{1}^{3}, S_{2}^{1}, S_{2}^{2}, S_{2}^{3}$ представимо абстрактными конечными инициальными автоматами с входным алфавитом $A$, выходным алфавитом $B$ и алфавитами состояний мощности не более $Q$.

Применим лемму о сокращении для слов $s_{1}^{1}=s_{2}^{1}=\alpha$ с общей частью $\alpha$. По ней существует $\alpha^{\prime} \in S_{1}^{1} \cap S_{2}^{1}:\left|\alpha^{\prime}\right| \leqslant Q^{2}$ : 


\begin{tabular}{|c|c|c|c|c|c|c}
\hline$\alpha_{1}$ & $s_{1}^{2}$ & $s_{1}^{3}$ & $m_{1}^{1}$ & $m_{1}^{2}$ & $m_{1}^{3}$ & \\
\cline { 2 - 7 } & $a_{1}$ & \multicolumn{6}{|c}{$\tilde{\beta}$} \\
\hline
\end{tabular}

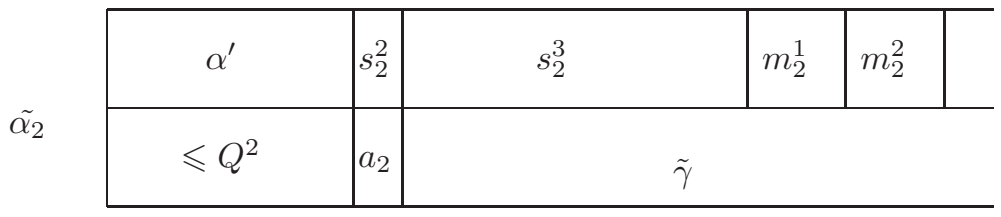

рис. 5

В образе $\tilde{f}\left(\tilde{\alpha_{1}}\right)=\tilde{f}\left(\tilde{\alpha_{2}}\right)$ получаем:

\begin{tabular}{|c|c|c|c|c|c|c}
\hline$\alpha_{1}$ & $s_{1}^{2}$ & $s_{1}^{3}$ & $m_{1}^{1}$ & $m_{1}^{2}$ & $m_{1}^{3}$ & \\
\cline { 2 - 6 } & $\leqslant Q^{2}$ & $a_{1}$ & \multicolumn{3}{|c}{$\tilde{\beta}$} \\
\hline
\end{tabular}

верхнее разбиение

\begin{tabular}{|c|c|c|c|c|c|c|}
\hline$\tilde{f}\left(\alpha^{\prime}\right)$ & $\tilde{f}\left(a_{1}\right)$ & $\tilde{f}\left(s_{1}^{3}\right)$ & $\tilde{f}\left(m_{1}^{1}\right)$ & $\tilde{f}\left(m_{1}^{2}\right)$ & $\tilde{f}\left(m_{1}^{3}\right)$ \\
\hline$\tilde{f}$ & $\tilde{f}\left(\alpha^{\prime}\right)$ & $\tilde{f}\left(a_{2}\right)$ & $\tilde{f}\left(s_{2}^{3}\right)$ & $\tilde{f}\left(m_{2}^{1}\right)$ & $\tilde{f}\left(m_{2}^{2}\right)$ & \\
\hline
\end{tabular}

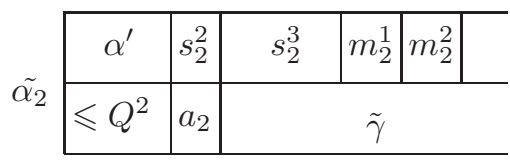

нижнее разбиение

рис. 6

По лемме об алфавитной детерминизации слова $\tilde{f}\left(\alpha^{\prime}\right), \tilde{f}\left(a_{1}\right), \tilde{f}\left(s_{1}^{3}\right), \tilde{f}\left(m_{1}^{1}\right), \ldots, \tilde{f}\left(a_{2}\right)$, $\tilde{f}\left(s_{2}^{3}\right), \tilde{f}\left(m_{2}^{1}\right), \ldots$ из образа на рис. 6 распознаются абстрактными конечными инициальными автоматами с входным алфавитом $B$ с не более чем $\tilde{Q}$ состояниями (для каждого слова своим).

Обозначим через $\beta$ произвольное из слов верхнего разбиения (см. рис. 6). Оно распознается абстрактным конечным инициальным автоматом $V$ с не более чем $\tilde{Q}$ состояниями (см. рис. 6). Обозначим через $V^{-1}$ автомат с входным алфавитом $A$, из которого был получен автомат $V$ при применении леммы об алфавитной детерминизации. Если слово $\beta$ разбивается при измельчении нижним разбиением на более чем $\tilde{Q}+3$ подслов, то три из них могут быть частями слов $\tilde{f}\left(\alpha^{\prime}\right), \tilde{f}\left(a_{2}\right), \tilde{f}\left(s_{2}^{3}\right)$, а оставшиеся будут словами из множества $\left\{\tilde{f}\left(m_{2}^{i}\right) \mid i \in \mathbb{N}\right\}$. Рассмотрим состояния, в которых окажется автомат $V$ при подаче на вход слова $\beta$ во время прохождения граничных точек измельчения: 
$\beta$

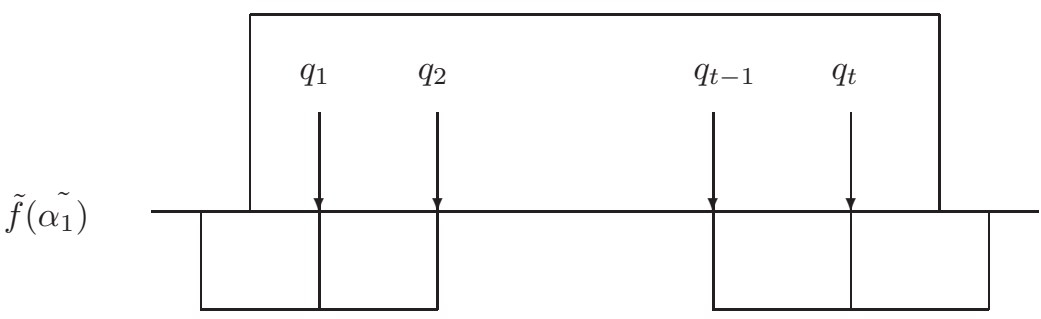

рис. 7

Здесь $t>\tilde{Q}+2$. После как минимум $\tilde{Q}+1$ состояний в нижнем разбиении начинаются куски из $\tilde{f}\left(m_{2}^{i}\right), i \in \mathbb{N}$. Так как в автомате $V$ не более $\tilde{Q}$ состояний, то по принципу Дирихле среди таких состояний найдется пара одинаковых. Если выкинуть подслово $\gamma$ слова $\beta$ между этими двумя позициями с совпадающими состояниями, то новое слово $\beta^{\prime}$ будет по-прежнему распознаваться автоматом $V$.

$\beta$

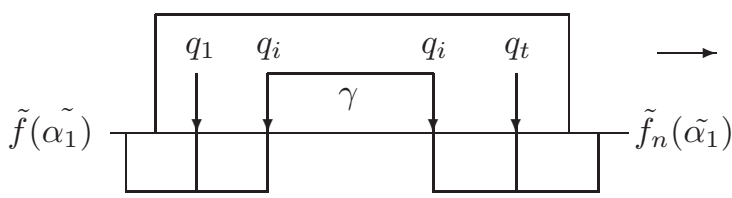

рис. 8

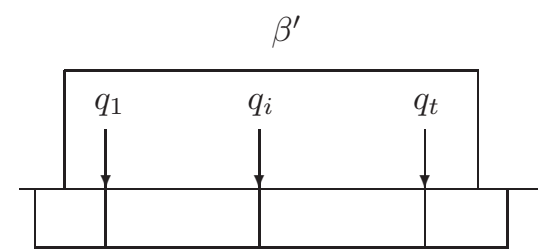

Обозначим слово, полученное после выкидывания из $\tilde{f}\left(\tilde{\alpha_{1}}\right)$ подслова $\gamma$, через $\tilde{f}_{n}\left(\tilde{\alpha}_{1}\right)$ (см. рис. 8). Так как слово $\beta^{\prime}$ распознается автоматом $V$, то существует слово $\alpha^{\prime}$ в алфавите $A$, распознаваемое автоматом $V^{-1}$. Из этого факта и леммы о разрезе следует, что слово $\tilde{f}_{n}\left(\tilde{\alpha}_{1}\right)$ лежит в $S_{1} \cdot\left(M_{1}\right)^{\infty}$. При выкидывании подслова $\gamma$ из нижнего разбиения выпадает несколько слов из множества $\left\{\tilde{f}\left(m_{2}^{i}\right) \mid i \in \mathbb{N}\right\}$. Значит, слово $\tilde{f}_{n}\left(\tilde{\alpha}_{1}\right)$ лежит и в $S_{2} \cdot\left(M_{2}\right)^{\infty}$. Новые верхнее и нижнее разбиения гарантируют наличие двух расшифровок слова $\tilde{f}_{n}\left(\tilde{\alpha}_{1}\right)$ в алфавите $A$. Эти расшифровки различны, так как в них на одном и том же месте, как и в сверхсловах $\tilde{\alpha}_{1}, \tilde{\alpha_{2}}$, стоят различные буквы $a_{1}$ и $a_{2}$. Поэтому можно изначально считать, что слово $\beta$ разбивается при измельчении нижним разбиением на не более чем $\tilde{Q}+3$ подслов. Далее, несколько раз применяя лемму о сокращении, добиваемся, чтобы все подслова $\beta^{\prime}$, являющиеся словами нижнего разбиения, имели длину не больше $\tilde{Q}^{2}$. Отсюда непосредственно получаем, что $|\beta| \leqslant \tilde{Q}^{2} \cdot(\tilde{Q}+3)$. Проведя аналогичные рассуждения для измельчения нижнего разбиения верхним, получаем, что и для произвольного слова $\beta$ из нижнего разбиения выполняется неравенство $|\beta| \leqslant \tilde{Q}^{2} \cdot(\tilde{Q}+3)$. Обозначим это число через $L(\tilde{Q})$.

Рассмотрим деве окончания $\tilde{f}\left(m_{1}^{1}\right) \cdot \tilde{f}\left(m_{1}^{2}\right) \cdot \tilde{f}\left(m_{1}^{3}\right) \ldots$ и $\tilde{f}\left(m_{2}^{1}\right) \cdot \tilde{f}\left(m_{2}^{2}\right) \cdot \tilde{f}\left(m_{2}^{3}\right) \ldots$ сверхслова $\tilde{f}\left(\tilde{\alpha_{1}}\right)=\tilde{f}\left(\tilde{\alpha_{2}}\right)$. По ним естественно строятся их разбиения на $\tilde{f}\left(m_{1}^{1}\right), \tilde{f}\left(m_{1}^{2}\right)$, $\tilde{f}\left(m_{1}^{3}\right), \ldots$ и $\tilde{f}\left(m_{2}^{1}\right), \tilde{f}\left(m_{2}^{2}\right), \tilde{f}\left(m_{2}^{3}\right), \ldots$ Возьмем измельчение этих двух разбиений на их общей части. Слова измельчения разделим на 2 группы. К первой группе отнесем слова, являющиеся собственным началом слова одного из разбиений и собственным окончанием слова другого разбиения. Ко второй группе отнесем слова, являющиеся словами одного из разбиений: 


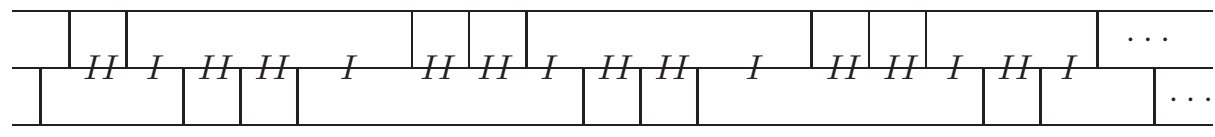

рис. 9

Каждое слово из первой группы имеет длину не больше $L(\tilde{Q})-1$. Найдем количество различных слов в алфавите $B$, длина которых не превосходит $L(\tilde{Q})-1$ :

$$
|B|+|B|^{2}+\ldots+|B|^{L(\tilde{Q})-1}=\frac{|B|^{L(\tilde{Q})}-|B|}{|B|-1} .
$$

Обозначим это число через $L(B, \tilde{Q})$. Если слово из первой группы встречается хотя бы три раза, то среди них найдутся два слова одной ориентации:

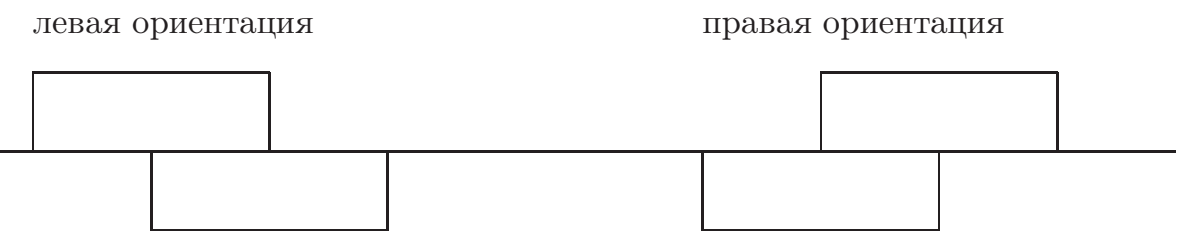

рис. 10

Два слова левой ориентации позволяют построить периодическое сверхслово с двумя разными разбиениями:

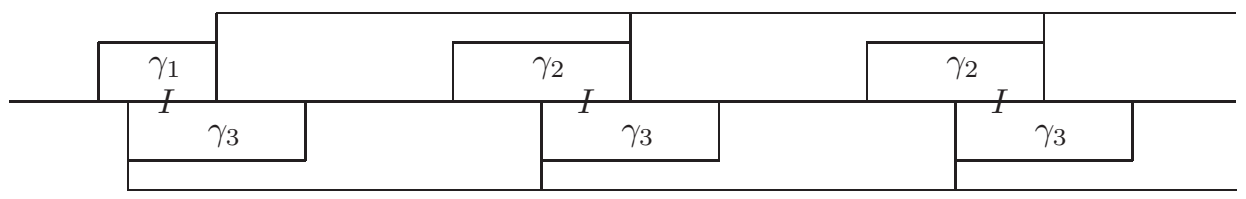

рис. 11

Для двух слов правой ориентации периодическое сверхслово строится аналогично. Заметим, что это сверхслово будет по-прежнему иметь две различные расшифровки из $S_{1} \cdot\left(M_{1}\right)^{\infty}$ и $S_{2} \cdot\left(M_{2}\right)^{\infty}$, так как в них на одном и том же месте, как и в сверхсловах $\tilde{\alpha_{1}}, \tilde{\alpha_{2}}$, будут стоять различные буквы $a_{1}$ и $a_{2}$.

В силу изложенного выше сумма длины предпериода и длины периода полученных таким образом слов в верхнем разбиении не превосходит

$$
\left|\alpha^{\prime}\right|+\left|a_{1}\right|+\left|s_{1}^{3}\right|+2 L(\tilde{Q}) L(B, \tilde{Q}) .
$$

Для нижнего разбиения получаем аналогичную оценку:

$$
\left|\alpha^{\prime}\right|+\left|a_{2}\right|+\left|s_{2}^{3}\right|+2 L(\tilde{Q}) L(B, \tilde{Q}) .
$$

Окончательная оценка для обоих разбиений равна:

$$
Q^{2}+1+L(\tilde{Q})+2 L(\tilde{Q}) L(B, \tilde{Q})
$$


Случай 2.

$l+1>\left|s_{1}\right|, l+1 \leqslant\left|s_{2}\right|$ (см. рис. 12).

\begin{tabular}{|c|c|c|c|c|}
\hline$s_{1}$ & $m_{1}^{1}$ & $m_{1}^{2}$ & \multicolumn{2}{|c|}{$m_{1}^{3}$} \\
\hline \multicolumn{3}{|c|}{$\alpha$} & $a_{1}$ & $\tilde{\beta}$ \\
\hline
\end{tabular}

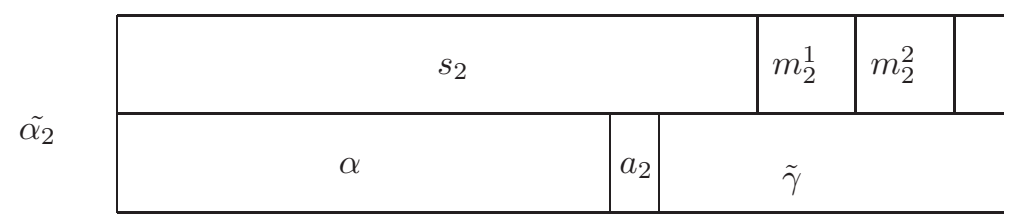

рис. 12

Без ограничения общности можно считать, что $a_{1} \in m_{1}^{3}$. Применим лемму о разрезе для слова $m_{1}^{3} \in M_{1}$ по местам $s:=l-\left|s_{1}\right|-\left|m_{1}^{1}\right|-\left|m_{1}^{2}\right|$ и $s:=l-\left|s_{1}\right|-\left|m_{1}^{1}\right|-$ $\left|m_{1}^{2}\right|+1$ и для слова $s_{2} \in S_{2}$ по местам $s:=l$ и $s:=l+1$ так, как это показано на рисунке 13.

\begin{tabular}{|c|c|c|c|c|c|}
\hline$s_{1}$ & $m_{1}^{1}$ & $m_{1}^{2}$ & $m_{1,1}^{3}$ & $a_{1}$ & $m_{1,3}^{3}$ \\
\hline \multicolumn{4}{|c|}{$\alpha$} & $a_{1}$ & $\tilde{\beta}$ \\
\hline
\end{tabular}

\begin{tabular}{|l|l|l|l|l|l}
\hline$s_{2}^{1}$ & $s_{2}^{2}$ & $s_{2}^{3}$ & $m_{2}^{1}$ & $m_{2}^{2}$ & \\
\cline { 2 - 6 }$\alpha_{2}$ & $a_{2}$ & \multicolumn{3}{|c}{$\tilde{\gamma}$} \\
\hline
\end{tabular}

рис. 13

Отличие рассуждений от случая 1 состоит в том, что к итоговой оценке добавляется оценка длины подслова $m_{1}^{1} \cdot m_{1}^{2} \cdot m_{1,1}^{3}$. Каждое из слов $m_{1}^{i}$ распознается одним и тем же автоматом, задающим $M_{1}$. Обозначим этот автомат через $V_{1}$. Теперь применим идею леммы о сокращении к словам $m_{1}^{1} \cdot m_{1}^{2} \cdot m_{1,1}^{3}$ и $s_{2}^{1}$. Тонкость состоит в том, что границе между $m_{1}^{1}$ и $m_{1}^{2}$, равно как и границе между $m_{1}^{2}$ и $m_{1,1}^{3}$, ставится в соответствие начальное состояние автомата $V_{1}$. K общей оценке добавится $Q^{2}$. Таким образом, итоговая оценка равна:

$$
2 Q^{2}+1+L(\tilde{Q})+2 L(\tilde{Q}) L(B, \tilde{Q}) .
$$


Случай 3.

$l+1 \leqslant\left|s_{1}\right|, l+1>\left|s_{2}\right|$.

Случай сводится к случаю 2 перестановкой сверхслов $\tilde{\alpha_{1}}$ и $\tilde{\alpha_{2}}$.

Случай 4.

$l+1>\left|s_{1}\right|, l+1>\left|s_{2}\right|$.

\begin{tabular}{|c|c|c|c|c|}
\hline$s_{1}$ & $m_{1}^{1}$ & $m_{1}^{2}$ & \multicolumn{2}{|c|}{$m_{1}^{3}$} \\
\hline \multicolumn{3}{|c|}{$\alpha$} & $a_{1}$ & $\tilde{\beta}$ \\
\hline
\end{tabular}

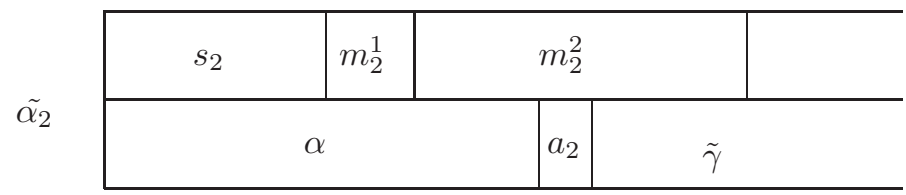

рис. 14

Без ограничения общности можно считать, что $\left|s_{1}\right| \leqslant\left|s_{2}\right|, a_{1} \in m_{1}^{3}$ и $a_{2} \in m_{2}^{2}$. После применения леммы о разрезе получаем:

\begin{tabular}{|c|c|c|c|c|c|}
\hline$s_{1}$ & $m_{1}^{1}$ & $m_{1}^{2}$ & $m_{1,1}^{3}$ & $a_{1}$ & $m_{1,3}^{3}$ \\
\hline \multicolumn{4}{|c|}{$\alpha$} & $a_{1}$ & $\tilde{\beta}$ \\
\hline
\end{tabular}

\begin{tabular}{|l|l|l|l|l|l}
\hline$s_{2}$ & $m_{2}^{1}$ & $m_{2,1}^{2}$ & $a_{2}$ & $m_{2,3}^{2}$ & \\
\cline { 2 - 6 }$\alpha$ & & $a_{2}$ & \multicolumn{2}{|c}{$\tilde{\gamma}$} \\
\hline \multicolumn{2}{c|}{$\alpha$} \\
\hline
\end{tabular}

рис. 15

Отличие рассуждений от случая 1 состоит в том, что к итоговой оценке добавляются две оценки. Одна - оценка длины общей части слов $m_{1}^{1} \cdot m_{1}^{2} \cdot m_{1,1}^{3}$ и $\left|s_{2}\right|$. Вторая - длины общей части слов $m_{1}^{1} \cdot m_{1}^{2} \cdot m_{1,1}^{3}$ и $m_{2}^{1} \cdot m_{2,1}^{2}$. Получение этих оценок повторяет получение оценки из случая 2 . K общей оценке добавится $2 Q^{2}$. Таким 
образом, итоговая оценка имеет вид

$$
3 Q^{2}+1+L(\tilde{Q})+2 L(\tilde{Q}) L(B, \tilde{Q}) .
$$

Разбор случаев закончен. Подставляя значения для $L(\tilde{Q})$ и $L(B, \tilde{Q})$, получаем требуемую оценку. Утверждение леммы доказано.

Заключительная лемма. Существует алгоритм, определяющий по произвольной паре $(\tilde{f}, \tilde{R})$, где $\tilde{f}$ - алфавитное кодирование из алфавита $A$ в алфавит $B$, а $\tilde{R}$ - общерегулярное сверхсобытие в алфавите $A$, существуют ли $\tilde{\alpha}_{1}, \tilde{\alpha}_{2} \in \tilde{R}$, $\tilde{\alpha_{1}} \neq \tilde{\alpha_{1}}$, для которых выполняется $\tilde{f}\left(\tilde{\alpha_{1}}\right)=\tilde{f}\left(\tilde{\alpha_{1}}\right)$.

Доказательство. По лемме о сверхсобытии существуют такие регулярные события $S_{1}, M_{1}, \ldots, S_{k}, M_{k}$ в алфавите $A$, что $R=\bigcup_{i=1}^{k} S_{i} \cdot\left(M_{i}\right)^{\infty}$. По теореме Клини эти события представимы некоторыми абстрактными конечными инициальными автоматами с входным алфавитом $A$ и выходным алфавитом $B$. Их количество конечно, поэтому существует такое натуральное число $Q$, что мощности алфавитов состояний этих автоматов не превосходят $Q$. Пусть

$$
\begin{gathered}
\tilde{Q}:=2^{Q+Q|A|(|f|-1)}, \quad L(B, \tilde{Q}):=\frac{|B|^{L(\tilde{Q})}-|B|}{|B|-1}, \\
L(B, Q, \tilde{Q}):=3 Q^{2}+1+L(\tilde{Q})+2 L(\tilde{Q}) L(B, \tilde{Q}) .
\end{gathered}
$$

По лемме о начале сверхсобытия существует алгоритм, который строит множество $M(L(B, Q, \tilde{Q}), \tilde{R})$. По этому множеству можно конечным перебором определить, верно ли, что для некоторых $1 \leqslant j \leqslant k, 1 \leqslant h \leqslant k$ существуют слова $\alpha_{1} \in S_{j}, \alpha_{2} \in S_{h}$, $\beta_{1} \in M_{j}^{*}, \beta_{2} \in M_{h}^{*}$, удовлетворяющие условиям

1) $\alpha_{1} \cdot\left(\beta_{1}\right)^{\infty} \neq \alpha_{2} \cdot\left(\beta_{2}\right)^{\infty}$,

2) $\tilde{f}\left(\alpha_{1} \cdot\left(\beta_{1}\right)^{\infty}\right)=\tilde{f}\left(\alpha_{2} \cdot\left(\beta_{2}\right)^{\infty}\right)$,

3) $\max \left(\left|\alpha_{1}\right|+\left|\beta_{1}\right|,\left|\alpha_{2}\right|+\left|\beta_{2}\right|\right) \leqslant L(B, Q, \tilde{Q})$.

Если такие слова есть, то $\tilde{f}$ не однозначно декодируемо на $\tilde{R}$. Если таких слов нет, то по главной лемме $\tilde{f}$ однозначно декодируемо на $\tilde{R}$. Алгоритм проверки однозначности алфавитного декодирования, определяющий по произвольной паре $(\tilde{f}, \tilde{R})$, однозначно ли декодируемо $\tilde{f}$ на $\tilde{R}$, построен. Утверждение леммы доказано.

Нетрудно видеть, что из заключительной леммы вытекает утверждение Теоремы 1.

\section{4. Дополнение}

Покажем, что проблему однозначности алфавитного декодирования общерегулярных сверхязыков нельзя тривиально свести к проблеме однозначности алфавитного декодирования регулярных языков.

Утверждение 1. Существуют такие алфавиты $A$ и $B$, алфавитное кодирование $\tilde{f}$ из алфавита $A$ в алфавит $B$ и общерегулярное сверхсобытие $\tilde{R}$ в алфавите $A$, что $\tilde{f}$ однозначно декодируемо на $\bigcup_{k=1}^{\infty} M(k, \tilde{R})$, но не однозначно декодируемо на $\tilde{R}$. 
Доказательство. Пусть $A=\left\{a_{1}, a_{2}, a_{3}\right\}, B=\left\{b_{1}, b_{2}\right\}, \tilde{f}\left(a_{1}\right):=b_{1}, \tilde{f}\left(a_{2}\right):=b_{1} b_{2}$, $\tilde{f}\left(a_{3}\right):=b_{2} b_{2}$. В качестве $\tilde{R}$ возьмем произвольное общерегулярное сверхсобытие в алфавите $A$, содержащее сверхслова $a_{1} a_{3}^{\infty}$ и $a_{2} a_{3}^{\infty}$. Например, $\tilde{R}:=\left\{a_{1}\right\} \cdot\left(\left\{a_{3}\right\}\right)^{\infty} \cup$ $\left\{a_{2}\right\} \cdot\left(\left\{a_{3}\right\}\right)^{\infty}$. Тогда $\tilde{f}\left(a_{1} a_{3}^{\infty}\right)=\tilde{f}\left(a_{2} a_{3}^{\infty}\right)=a_{1} b_{1}^{\infty}$. Значит, $\tilde{f}$ не однозначно декодируемо на $\tilde{R}$.

Пусть $\tilde{f}\left(\alpha_{1}\right)=\tilde{f}\left(\alpha_{2}\right)$ для некоторых $\alpha_{1}, \alpha_{2} \in A^{*}$. Возможны 3 случая.

1) $\left.]_{1}\left(\tilde{f}\left(\alpha_{1}\right)\right)=\right]_{1}\left(\tilde{f}\left(\alpha_{2}\right)\right)=b_{1}$. Тогда $\left.]_{1}\left(\alpha_{1}\right)=\right]_{1}\left(\alpha_{2}\right)=a_{1}$.

2) $\left.]_{2}\left(\tilde{f}\left(\alpha_{1}\right)\right)=\right]_{2}\left(\tilde{f}\left(\alpha_{2}\right)\right)=b_{1} b_{2}$. Тогда $\left.]_{1}\left(\alpha_{1}\right)=\right]_{1}\left(\alpha_{2}\right)=a_{2}$.

3) $\left.]_{2}\left(\tilde{f}\left(\alpha_{1}\right)\right)=\right]_{2}\left(\tilde{f}\left(\alpha_{2}\right)\right)=b_{2} b_{2}$. Тогда $\left.]_{1}\left(\alpha_{1}\right)=\right]_{1}\left(\alpha_{2}\right)=a_{3}$.

Во всех случаях $\left.]_{1}\left(\alpha_{1}\right)=\right]_{1}\left(\alpha_{2}\right)$. Повторяя рассуждение конечное число раз, получаем: $\alpha_{1}=\alpha_{2}$. Значит, $\tilde{f}$ однозначно декодируемо на $A^{*}$, а значит, и на $\bigcup_{k=1}^{\infty} M(k, \tilde{R})$. Утверждение доказано.

\section{Список литературы}

1. Кудрявцев В. Б., Алешин С. В., Подколзин А. С., Введение в теорию автоматов. Наука, Москва, 1985.

2. С. В. Яблонский, Введение в дискретную математику. Наука, Москва, 1986.

3. Дергач П. С., Об однозначности алфавитного декодирования. Интеллектуальные системы (2011) 15, №3, 349-360.

Статья поступила 19.02.2013. 